- remove the link when the scam was uncovered. The action triggered "a barrage of complaints and requests to reactivate the link from representatives of the false journal", says Marie McVeigh, director of content selection at Thomson Reuters. She says that the company has also received enquiries from customers "asking why the articles that had been accepted by one of the false journals were not appearing in our indexes".

"The quality and integrity of our content is of the greatest importance to us," adds McVeigh.

In a further impudent touch, the various counterfeit Archives des Sciences websites list an editorial board with 87 members, including Daniel Gamelin, a chemist and materials scientist at the University of Washington in Seattle, and Gerald Cleaver, a high-energy physicist at Baylor University in Waco, Texas. Both are perplexed and annoyed. "This is the first I have heard of this website or of my listing; I have no affiliation with this organization, nor have I ever," says Gamelin. Cleaver, too, says that his name is being used without his permission.

The 'editor-in-chief' of the fake Archives des Sciences journal is named on the counterfeit websites as "Prof. Dr. Eliana Schmid", with the affiliation "Geneva, Switzerland". The counterfeit Wulfenia sites give as the editor-in-chief Vienna S. Franz and list 35 editorial-board members, with most affiliations giving only city and country. Eberwein and Degli Agosti think that these named editors-in-chief are fictional.

Researchers who have submitted to the fake journals pay dearly. Both the counterfeit Archives des Sciences and the fake Wulfenia charge author fees of more than $\$ 500$, with instructions to address payment to accounts at two banks in Yerevan, Armenia.

Degli Agosti has reported the counterfeit Archives des Sciences websites to the Cybercrime Coordination Unit Switzerland, but was told that the sites were hosted in the United States, so the unit could not act directly against them. However, it advised him that he could press criminal charges under Swiss cybercrime laws. The University of Geneva's lawyers are helping the SPHN to draft its case, but the society is not part of the university, so it will have to take its case forward alone.

Austrian police have made little progress on the Wulfenia case, says Eberwein: they closed down a fake site hosted in Austria, but multiple replicas popped up on servers in other countries. Austrian authorities have told Eberwein that the scope for legal redress is limited, but, unconvinced, he has contacted Archives des Sciences to swap notes. $n$ SEE NEWS FEATURE P.433

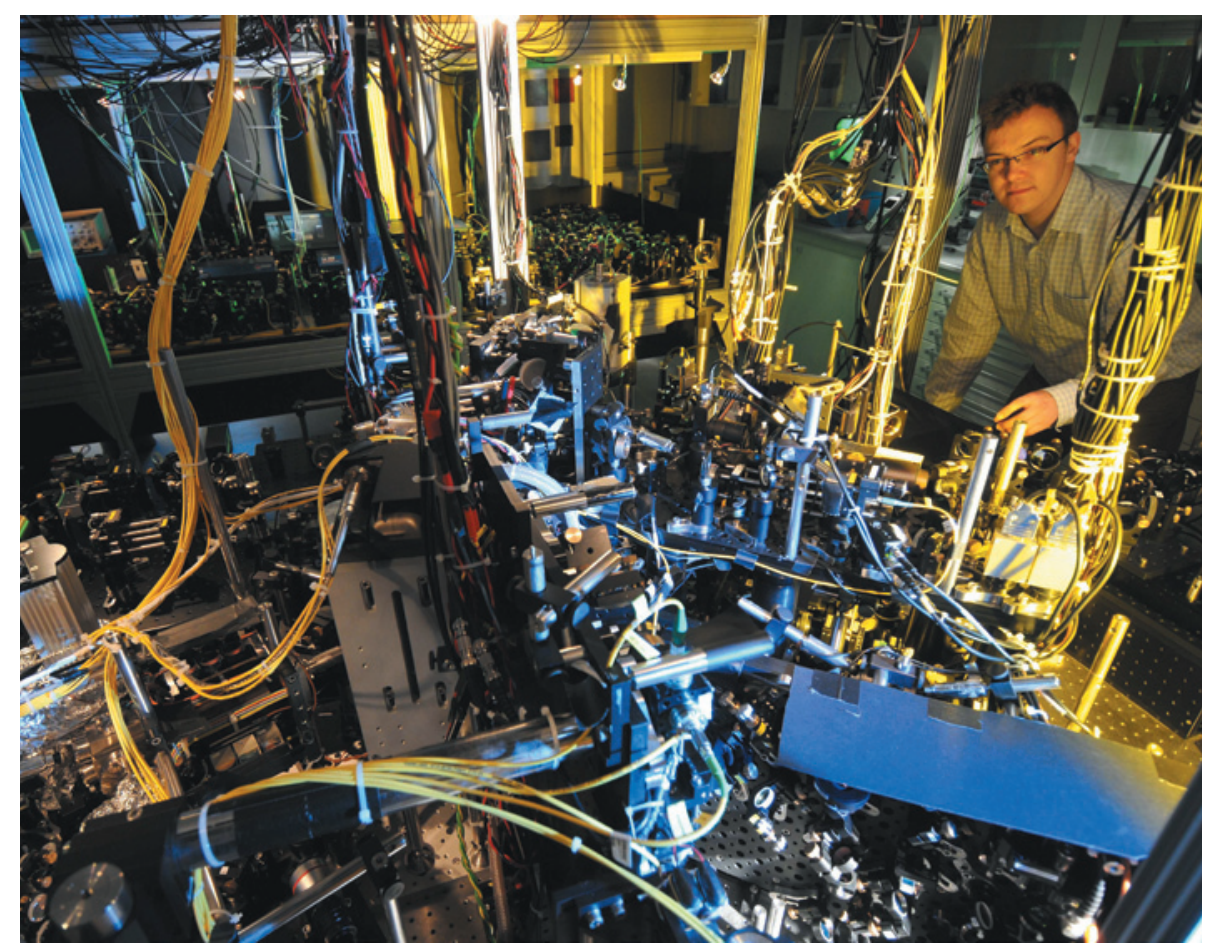

Physicists have found Higgs-like particles in a superfluid at the Max Planck Institute in Munich, Germany.

\title{
PHYSICS
}

\section{Higgs physics on the cheap}

\section{Tabletop Higgs particles may illuminate cosmic cousin.}

\section{BY EUGENIE SAMUEL REICH}

$\mathrm{M}$ ore than three decades ago, before the world's most powerful particle collider was even on the drawing board, two physicists discovered a Higgs boson. On a tabletop.

In 1981, Peter Littlewood and Chandra Varma, two solid-state theorists at Bell Laboratories in Murray Hill, New Jersey, realized that a mysterious effect seen in a niobium selenide superconductor could be explained by the jiggling of the invisible field that causes electrons in the material to pair up and move as one without resistance. Mathematically, the disturbance in the field looked very like one that is associated with the Higgs particle found by particle physicists.

Because the superconducting field was already known, the search for the associated particle never drew as much attention as the hunt by particle physicists for the Higgs. That quest culminated on 13 March at the Large Hadron Collider (LHC) at CERN, Europe's main particle-physics facility near Geneva

in Switzerland, with the announcement of a definitive Higgs detection: the first direct evidence of a Higgs field permeating the Universe and giving objects mass.

But physicists agree that the superconducting Higgs is closely related to its particlephysics cousin. Both arise from the vibration of an invisible field that forces ordinary particles to oscillate in sync. Now physicists are seeing signs that other condensed-matter systems can generate Higgs-like particles, raising hopes that work on one Higgs, studied cheaply on tabletops, can inform the study of another, probed by a US $\$ 5$-billion collider. "I'm hoping there will be cross-fertilization," says Varma, now at the University of California, Riverside, who spoke in a packed session on solid-state Higgs particles at a meeting of the American Physical Society (APS) in Baltimore, Mary-

$\rightarrow$ NATURE.COM For CERN's discovery of the Higgs, see: go.nature.com/gmp3ef land, on 19 March.

It wouldn't be the first time that particle physics stood on the shoulder of condensed-matter physics. When Peter 


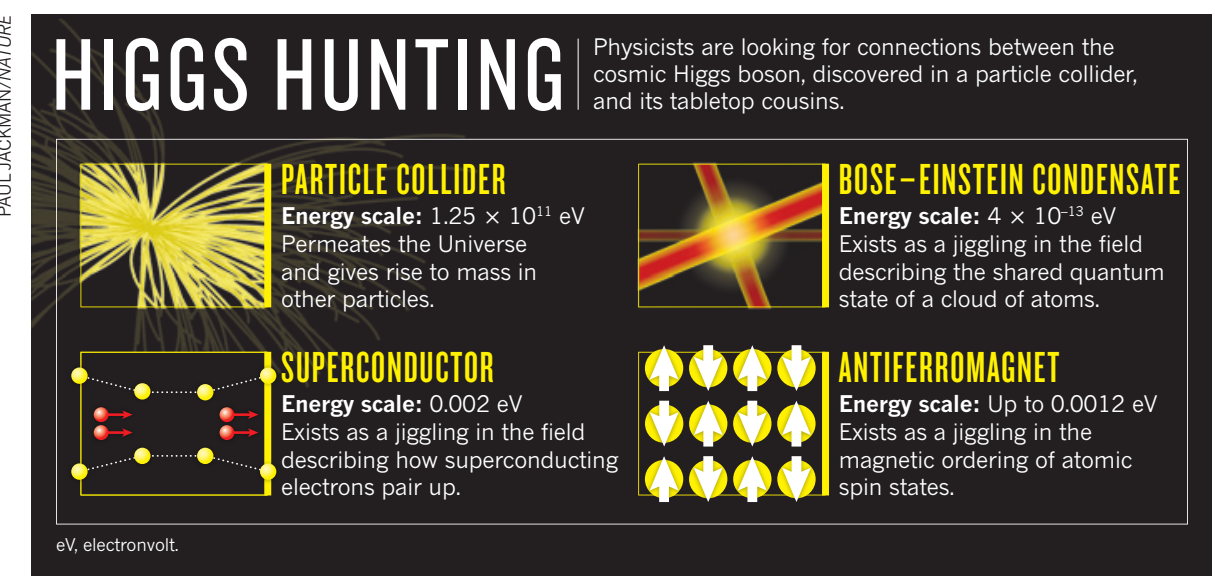

Higgs, a theorist at the University of Edinburgh, UK, put together the idea known as the Higgs field in 1964, he built on a theory developed a year earlier, by theorist Phil Anderson, now at Princeton University in New Jersey, to describe the interactions of superconducting electrons. Higgs took the idea further, interpreting the field as a medium in empty space that would pull on particles, giving them mass. The Higgs bosons that made up the field would be invisible unless the field was jiggled in the right way, as occurs in high-energy collisions at the LHC.

Varma and Littlewood identified their superconducting Higgs in the same way, by recognizing that a wave of slightly displaced atoms was jiggling the superconducting field. Now researchers are trying to extend their catalogue of Higgs particles. At the APS meeting, physicist Marie-Aude Méasson from the University of Paris Diderot and her colleagues reported that the mechanism by which the Higgs was recognized in niobium selenide did not exist in a related superconductor, niobium sulphide, which lacks the wave of displaced atoms. Méasson says that she wants to find examples of other superconductors that can produce Higgs particles. "When you only have one example in nature you wonder if it's really what you think," she says.

Other researchers are looking for examples outside superconductors. Christian Rüegg of the Paul Scherrer Institute in Villigen, Switzerland, says that his research group has produced Higgs-like particles in an antiferromagnet, in which the spins of atoms are opposite to adjacent ones. And physicist Immanuel Bloch of the Ludwig Maximilians University in Munich, Germany, told scientists at the APS meeting that he has created Higgs particles in a cloud of chilled rubidium atoms that share the same quantum state (a Bose-Einstein condensate). Using criss-crossing laser beams, Bloch tunes the cloud to a transition point between the atoms' insulating and superfluid states at which the Higgs particle emerges. Although the speed of light is a factor in equations describing the particle-physics Higgs, it is replaced by the speed of sound in Bloch's system, which means that the energy scale of the particles is vastly smaller than that of that of the cosmic Higgs (see 'Higgs hunting'). This mini-Higgs, says Bloch, is "a scaled-down version".

Research on such solid-state Higgs particles might illuminate future directions for particle physics, says Subir Sachdev, a physicist at Harvard University in Cambridge, Massachusetts, who organized the APS session. A major disappointment of the Higgs discovered at the LHC is its apparently 'plain vanilla' nature. Theorists had hoped for a composite Higgs that would have pointed to the existence of other undiscovered particles and forces. But so far the Higgs has behaved exactly as expected, revealing little about what might lie beyond the standard model of particle physics, thought to be incomplete because it doesn't include the force of gravity. One obstacle at the LHC is that any undiscovered particles might decay almost immediately after they are created, too quickly for them to be studied. But Bloch's version of a Higgs particle lasts for one six-hundredth of a second, which allows him to closely observe details of particle decays.

Particle theorist John Ellis of CERN agrees that the study of solid-state Higgs particles might shed light on what lies beyond the standard model. But he notes that ideas can flow in the other direction, too: the mathematical tools developed by particle physicists to study strong interactions between particles are already being used by condensed-matter physicists in the hope of explaining high-temperature superconductors, in which some think that yet more Higgs particles might be lurking. "Maybe particle physics has given something back," says Ellis.

\section{CORRECTION}

The News Feature 'Campus 2.0' (Nature 495, 160-163; 2013) wrongly said that Andrew Ng went public with Daphne Koller's machine-learning course - it was his course. Both this course and a database course each attracted 100,000 students not 60,000 as stated. 